\title{
Temperature-Dependent Hydration at Micellar Surface: Activation Energy Barrier Crossing Model Revisited
}

\author{
Rajib Kumar Mitra, Sudarson Sekhar Sinha, and Samir Kumar Pal* \\ Unit for Nanoscience and Technology, Department of Chemical, Biological, and Macromolecular Sciences, \\ S. N. Bose National Center for Basic Sciences, Block JD, Sector III, Salt Lake, Kolkata 700098, India
}

Received: March 22, 2007; In Final Form: May 5, 2007

\begin{abstract}
In recent years, the validity of the activation energy barrier crossing model at the micellar surface brings notable controversy (Sen, P.; Mukherjee, S.; Halder, A.; Bhattacharyya, K. Chem. Phys. Lett. 2004, 385, 357-361. Kumbhakar, M.; Goel, T.; Mukherjee, T.; Pal, H. J. Phys. Chem. B 2004, 108, 19246-19254.) in the literature. In order to check the validity of the model by time-resolved solvation of a probe fluorophore, a wider range of temperature must be considered. At the same time, spatial heterogeneity (solubilization) of the probe and structural perturbation of the host micelle should carefully be avoided, which was not strictly maintained in the earlier studies. We report here the solvation dynamics of 4-(dicyanomethylene)-2-methyl6(p-dimethylamino-styryl) $4 H$-pyran (DCM) in the SDS micelle at 298, 323, and $348 \mathrm{~K}$. The probe DCM is completely insoluble in bulk water in this wide range of temperature. The size of the micelle at different temperatures using the dynamic light scattering (DLS) technique is found to have insignificant change. The hydration number of the micelle, determined by sound velocity measurements, decreases with increasing temperature. Time-resolved fluorescence anisotropy reveals the retention of the probe in the micellar interface within the temperature range. The average solvation time decreases with increasing temperature. The result of the solvation study has been analyzed in the light of energetics of bound to free water conversion at a constant size and decreasing hydration number at the micellar surface. The solvation process at the micellar surface has been found to be the activation energy barrier crossing type, in which interfacially bound type water molecules get converted into free type molecules. We have calculated $E_{\mathrm{a}}$ to be $3.5 \mathrm{kcal} \mathrm{mol}^{-1}$, which is in good agreement with that obtained by molecular dynamics simulation studies.
\end{abstract}

\section{Introduction}

In self-organized molecular assemblies, like micelles, the water molecules at the surfaces of the micelles have properties different from those of bulk water. ${ }^{1-10}$ Both theoretical and experimental studies prove the existence of a very slow component in the dynamics of water in such restricted environments. The slow component decays in hundreds to thousands of picoseconds depending upon the systems involved, whereas pure water molecules exhibit the solvation dynamics in the subpicosecond time scale. ${ }^{11}$ The origin of this slow component is due to a dynamic equilibrium between the bound and free water molecules in which the water molecules at the interface are hydrogen-bonded with the polar head group of the micelle. ${ }^{7,8}$ Some of the water molecules are directly hydrogen-bonded to the polar head group of the surfactant molecules, and some are in either free or intermolecularly hydrogen-bonded among themselves. ${ }^{5,9}$ The equilibrium between bound and free water molecules is sensitive to the change in the microenvironment of the microheterogeneous systems, e.g., temperature, pressure, additives, and so forth, since it can induce changes in different physicochemical properties of micelles. Thus, it is important to study the effect of different external conditions on the solvation dynamics of water in a restricted environment.

Bagchi et al. ${ }^{4,5,10}$ made simulation studies in order to understand the temperature-dependent water dynamics at the surface of $\mathrm{Cs}$ - pendecafluorooctanoate micelles at 300 and 350

* Corresponding author. E-mail: skpal@bose.res.in.
$\mathrm{K}$. They found that the reorientational motions of water molecules around the micelles slow down as temperature is reduced from 350 to $300 \mathrm{~K}$, which is due to the formation of bridge hydrogen bonds that the water molecules form with polar head groups. Sen et al. ${ }^{12}$ studied the solvation dynamics of 4-aminopthalamide (4-AP) in TX-100 micelles at three different temperatures of 283,303 , and $323 \mathrm{~K}$ using picosecond-resolved fluorescence Stokes'shift measurement (instrument response function $($ IRF $) \sim 100 \mathrm{ps}$ ). They observed an overall acceleration of the dynamics of solvation with an increase in temperature. The slow component of solvation $\left(\tau_{\text {solvation }}\right)$ can be expressed as ${ }^{7,10,13}$

$$
1 / \tau_{\text {solvation }} \approx k_{\mathrm{bf}}=\frac{k_{\mathrm{b}} T}{h} \mathrm{e}^{-\Delta G_{b f} / R T}
$$

where $k_{\mathrm{bf}}$ is the rate constant for bound water-to-free water interconversion and $\Delta G_{\mathrm{bf}}^{0}$ is the corresponding free energy change associated with this interconversion. They used an activation energy crossing barrier type model and plotted $1 /\left\langle\tau_{\text {solvation }}\right\rangle$ against $1 / T$ to obtain a linear plot and calculated the corresponding activation energy $\left(E_{\mathrm{a}}\right)$ to be $\sim 9 \mathrm{kcal} \mathrm{mol}^{-1}$. From the linearity of such an Arrhenius type of plot, they concluded that the change in structure and the hydration number of micelles have a minor role in the observed temperature dependence of solvation dynamics.

In the same year, Kumbhakar et al. ${ }^{14}$ published their study on the temperature dependency of the solvation dynamics of coumarin 153 (C-153) and coumarin 151 in TX-100 and Brij- 
35 micelles at temperatures 288, 298, and $308 \mathrm{~K}(\mathrm{IRF}=240$ ps). They found that while temperature change has a significant effect on the solvation dynamics of the TX-100 micellar system, the effect is nominal for the Brij-35 system. They found an unusual inversion of solvation time at $298 \mathrm{~K}$ for both the micellar systems. Particularly in the TX-100 micelle, the average solvation time constants at temperatures 288,298 , and $308 \mathrm{~K}$ are found to be 1281, 1981, and 1044 ps, respectively. Previously, using the TCSPC technique with better time resolution $\left(\right.$ IRF $=40 \mathrm{ps}$ ), Hara et al. ${ }^{15}$ reported an average solvation time constant of the probe (C-153) in TX-100 micelles at $293 \mathrm{~K}$ to be $920 \mathrm{ps}$, which is not in close agreement with those reported by Kumbhakar et al. ${ }^{14}$ The irregular trend of the solvation dynamics with temperature reported by Kumbhakar et al. ${ }^{14}$ is attributed to the change in hydration number and size of the micelles. Their results ${ }^{14}$ do not support the activation energy barrier crossing type model as employed by Sen et al. ${ }^{12}$ They criticized the work of Sen et al. ${ }^{12}$ in view of the choice of the probe. According to them, since the probe 4-AP is highly hydrophilic in nature, it generally resides in the interfacial region and in the bulk and not in the interior of the palisade layer of the micelles. Thus, the probe was unable to sense any change due to the structure and hydration of the micelles. The same group has recently reported ${ }^{16}$ the temperature dependent solvation of coumarin dyes in aqueous block copolymer micelles in the temperature range of $293-313 \mathrm{~K}$ and interpreted their results in view of structural and hydration studies.

Although a thorough understanding of the effect of temperature on the solvation dynamics in microheterogeneous systems is strongly demanding, only a few reports ${ }^{17-20}$ are available in the literature. Such temperature-dependent studies in micellar systems are rare in the literature, and only the three studies mentioned earlier ${ }^{12,14,16}$ have been made so far to the best of our knowledge. These previous studies, however, evoke a conflicting situation of whether the temperature induced change in solvation dynamics is due to an activation energy barrier crossing or is due to the change in size and hydration of the micelles. Such discrepancies in the studies made so far have led us to initiate the present study in order to determine the effect of temperature on the solvation dynamics at the micellar surface and to recheck the validity of the barrier crossing model.

We chose SDS micelles since the effect of temperature on the structural integrity of the micelles has been found to be negligible (see DLS study below). The choice of DCM [4-(dicyanomethylene)-2-methyl-6( $p$-dimethylamino-styryl) $4 \mathrm{H}$ pyran] as the probe has its origin rooted in its no water solubility. The most probable location of it is within the micellar interface,,$^{21,22}$ since it is strongly recommended that a good probe of solvation dynamics should be stationary so that the solvation response comes purely from the surrounding solvents. ${ }^{23} \mathrm{~A}$ wider range of temperature from 298 to $348 \mathrm{~K}$ has been chosen to carry out the study, in comparison to the previous studies. ${ }^{12,14}$ The structural integrity of the SDS micelles in this range of temperature has been investigated using the dynamic light scattering (DLS) technique. A temperature-dependent timeresolved anisotropy study has been made to explore the location of the probe at different temperatures. The hydration number of the micelles at different temperatures has been determined using ultrasound velocity measurements. Our aims of this study are to understand the effect of temperature on the solvation dynamics of a probe at the micellar surface, that retains its structural integrity, and its probable correlation with the hydration of the micelle-water interfacial layer and to find the

\section{SCHEME 1: Molecular Structure of DCM}

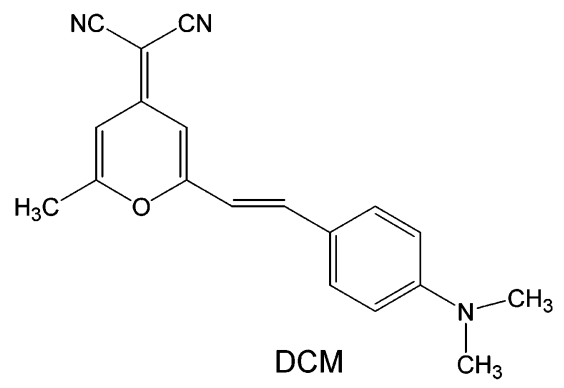

correctness of identifying the problem as an activation energy barrier crossing type.

\section{Materials and Methods}

Sodium dodecylsulfate (SDS) and 4-(dicyanomethylene)-2methyl-6(p-dimethylamino-styryl) $4 H$-pyran (DCM) [Scheme 1] are products of Fluka and Exciton, respectively, and are used without further purification. Solutions of $50 \mathrm{mM}$ SDS are prepared in $50 \mathrm{mM} \mathrm{NaCl}$ aqueous solution in order to avoid aggregation and used throughout the present study. Temperaturedependent steady-state emission is measured with a Jobin Yvon model Fluoromax-3 fluorimeter with a temperature controller attachment from Julabo (model: F32). DLS measurements are done with a Nano S Malvern instrument employing a $4 \mathrm{~mW}$ $\mathrm{He}-\mathrm{Ne}$ laser $(\lambda=632.8 \mathrm{~nm})$ equipped with a thermostatted sample chamber. All the scattered photons are collected at a $173^{\circ}$ scattering angle. The scattering intensity data are processed using the instrumental software to obtain the hydrodynamic diameter $\left(d_{\mathrm{H}}\right)$ and the size distribution of the scatterer in each sample. The instrument measures the time-dependent fluctuation in the intensity of light scattered from the particles in solution at a fixed scattering angle. The hydrodynamic diameter $\left(d_{\mathrm{H}}\right)$ of the micelles is estimated from the intensity auto-correlation function of the time-dependent fluctuation in intensity. $d_{\mathrm{H}}$ is defined as

$$
d_{\mathrm{H}}=\frac{k_{\mathrm{B}} T}{3 \pi \eta D}
$$

where $k_{\mathrm{B}}$ is the Boltzmann constant, $\eta$ is the viscosity, and $D$ is the translational diffusion coefficient. In a typical size distribution graph from the DLS measurement, the $x$-axis shows a distribution of size classes in nanometers, while the $y$-axis shows the relative intensity of the scattered light. This is therefore known as an intensity distribution graph.

The hydration number $\left(n_{\mathrm{h}}\right)$ of SDS micelles has been calculated using the density and sound velocity values measured by a densitymeter, model DSA5000 (density accuracy $10^{-6} \mathrm{~g}$ $\mathrm{cm}^{-3}$ ) from Anton Parr (Austria). The hydration number $\left(n_{\mathrm{h}}\right)$ of each SDS molecule is calculated as according to the model used by Bockris et al. ${ }^{24}$ and Rohman et al., ${ }^{25}$

$$
n_{\mathrm{h}}=\frac{n_{\mathrm{W}}}{n_{\mathrm{SDS}}}\left(1-\frac{\beta_{\mathrm{SDS}}}{\beta_{\mathrm{w}}} \frac{V}{N_{\mathrm{w}} \overline{V_{\mathrm{w}}}}\right)
$$

where the compressibility of a solution $(\beta)$ is calculated using the formula

$$
\beta=\frac{1}{\rho v^{2}}
$$

where $\rho$ is the density of the solution, $v$ is the velocity of sound through the solution, $\beta_{\mathrm{w}}$ and $\beta_{\mathrm{SDS}}$ are the compressibilities of 
water and the SDS micellar solution, respectively, $n_{\mathrm{w}}$ is the number of water molecules, $n_{\mathrm{SDS}}$ is the number of SDS molecules, $V$ is the total volume, $N_{\mathrm{w}}$ is the number of moles of water in the solution, and $\bar{V}_{\mathrm{w}}$ is the molar volume of water. Since the concentration of SDS is very low in the solution (50 $\mathrm{mM})$ as compared to that of water, $V$ can be taken to be equal to $N_{\mathrm{w}} \overline{V_{\mathrm{w}}}$, and eq 4 takes up the form

$$
n_{\mathrm{h}}=\frac{n_{\mathrm{W}}}{n_{\mathrm{SDS}}}\left(1-\frac{\beta_{\mathrm{SDS}}}{\beta_{\mathrm{W}}}\right)
$$

The number of water molecules and the number of SDS molecules can be replaced by the concentration $(c)$ of the species in the solution, and the final form of the equation is

$$
n_{\mathrm{h}}=\frac{c_{\mathrm{W}}}{c_{\mathrm{SDS}}}\left(1-\frac{\beta_{\mathrm{SDS}}}{\beta_{\mathrm{W}}}\right)
$$

Fluorescence transients have been measured and fitted by using the commercially available spectrophotometer (LifeSpec-ps) from Edinburgh Instrument, U.K. (excitation wavelength 409 $\mathrm{nm}, 80 \mathrm{ps}$ instrument response function) with an attachment for temperature-dependent studies (Julabo, model F32). The observed fluorescence transients are fitted by using a nonlinear least-square fitting procedure to a function $\left(X(t)=\int_{0}^{t} E\left(t^{\prime}\right) R(t\right.$ $\left.\left.-t^{\prime}\right) \mathrm{d} t^{\prime}\right)$ comprising of the convolution of the IRF $(E(t))$ with a sum of exponentials $\left(R(t)=A+\sum_{i=1}^{N} B_{i} \mathrm{e}^{-t / \tau_{i}}\right)$ with preexponential factors $\left(B_{i}\right)$, characteristic lifetimes $\left(\tau_{i}\right)$, and a background $(A)$. Relative concentration in a multiexponential decay is finally expressed as $a_{n}=B_{n} /\left(\sum_{i=1}^{N} B_{i}\right)$. The quality of the curve fitting is evaluated by reduced $\chi^{2}$ and residual data.

To construct time-resolved emission spectra (TRES), we follow the technique described in references. ${ }^{26,27}$ As described above, the emission intensity decays are analyzed in terms of the multiexponential model,

$$
I(\lambda, t)=\sum_{i=1}^{N} \alpha_{i}(\lambda) \exp \left[-t / \tau_{i}(\lambda)\right]
$$

where $\alpha_{i}(\lambda)$ are the pre-exponential factors, with $\sum \alpha_{i}(\lambda)=1.0$. In this analysis, we compute a new set of intensity decays, which are normalized so that the time-integrated intensity at each wavelength is equal to the steady-state intensity at that wavelength. Considering $F(\lambda)$ to be the steady-state emission spectrum, we calculate a set of $H(\lambda)$ values using

$$
H(\lambda)=\frac{F(\lambda)}{\int_{0}^{\infty} I(\lambda, t) \mathrm{d} t}
$$

which for multiexponential analysis becomes

$$
H(\lambda)=\frac{F(\lambda)}{\sum_{i} \alpha_{i}(\lambda) \tau_{i}(\lambda)}
$$

Then, the appropriately normalized intensity decay functions are given by

$$
I^{\prime}(\lambda, t)=H(\lambda) I(\lambda, t)=\sum_{i=1}^{N} \alpha_{i}^{\prime}(\lambda) \exp \left[-t / \tau_{i}(\lambda)\right]
$$
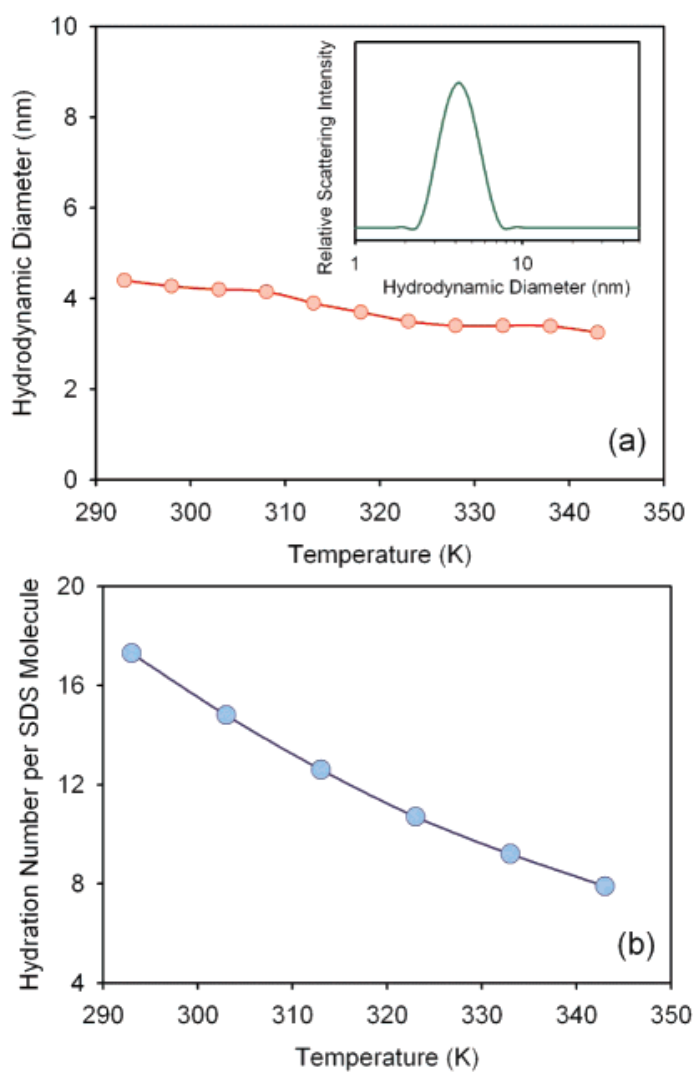

Figure 1. (a) Hydrodynamic diameter of $50 \mathrm{mM}$ SDS micelles at different temperatures as obtained from the dynamic light scattering (DLS) study. A typical DLS signal at $298 \mathrm{~K}$ has been depicted in the inset. The solid line is a guide for the eye. (b) Hydration number of 50 $\mathrm{mM}$ SDS micelles at different temperatures. The solid line is a guide for the eye.

where $\alpha_{i}^{\prime}(\lambda)=H(\lambda) \alpha_{i}(\lambda)$. The values of $I^{\prime}(\lambda, t)$ are used to calculate the intensity at any wavelength and time and thus the TRES. The values of the emission maxima and spectral width are determined by nonlinear least-square fitting of the spectral shape of the TRES. The spectral shape is assumed to follow a lognormal line shape, ${ }^{27}$

$$
I(\bar{v})=I_{0} \exp \left\{-\left[\ln 2\left(\frac{\ln (\alpha+1)}{b}\right)^{2}\right]\right\}
$$

with $\alpha=2 b\left(\bar{v}-\overline{v_{\max }}\right) / b>-1$, where $I_{0}$ is amplitude, $\bar{v}_{\max }$ is the wavenumber of the emission maximum, and the spectral width is given by $\Gamma=\Delta[\sinh (b) / b]$. The terms $b$ and $\Delta$ are asymmetry and width parameters. Equation 11 reduces to a Gaussian function for $b=0$. For anisotropy $(r(t))$ measurements, emission polarization is adjusted to be parallel or perpendicular to that of the excitation and the anisotropy is defined as $r(t)=$ $\left[I_{\text {para }}-\left(G I_{\text {perp }}\right)\right] /\left[I_{\text {para }}+\left(2 G I_{\text {perp }}\right)\right] . G$, the grating factor, is determined following the longtime tail matching technique. ${ }^{28}$

\section{Results and Discussion}

Figure 1a depicts the variation of the hydrodynamic diameter of $50 \mathrm{mM}$ SDS micelles as a function of temperature. A typical DLS signal at $298 \mathrm{~K}$ has been depicted in the inset of Figure 1a. The signal has been found to be highly monodispersed, and the measured hydrodynamic diameter of $4.3 \pm 0.4 \mathrm{~nm}$ is consistent with previous studies. ${ }^{29}$ It is evidenced from the figure that with an increase in temperature, the micellar diameter changes marginally. The high monodispersity of the DLS signal is retained in all the studied temperatures (figures not shown). 
TABLE 1: Density, Sound Velocity, and Hydration Number of $50 \mathrm{mM}$ SDS Solution

\begin{tabular}{|c|c|c|c|}
\hline temperature $(\mathrm{K})$ & density $\left(\mathrm{g} \mathrm{cm}^{-3}\right)$ & $\begin{array}{l}\text { sound velocity } \\
\qquad\left(\mathrm{m} \mathrm{s}^{-1}\right)\end{array}$ & $\begin{array}{c}\text { hydration } \\
\text { number per } \\
\text { SDS molecule }\end{array}$ \\
\hline 293 & 1.0044 & 1489.7 & 17.3 \\
\hline 303 & 1.0016 & 1514.9 & 14.8 \\
\hline 313 & 0.9980 & 1533.5 & 12.6 \\
\hline 323 & 0.9937 & 1546.1 & 10.7 \\
\hline 333 & 0.9888 & 1553.6 & 9.2 \\
\hline 343 & 0.9833 & 1556.4 & 7.9 \\
\hline
\end{tabular}

${ }^{a}$ Uncertainty in the measurement is $\pm 8 \%$.

The diameter decreases from $4.3 \mathrm{~nm}$ at $293 \mathrm{~K}$ to $3.4 \mathrm{~nm}$ at 343 K. It could be noted that for the nonionic surfactant (TX-100) used in the previous studies, the radius increases substantially on increasing the temperature. ${ }^{30}$ Thus in the present study, the structural integrity of the SDS micelles in the experimental temperature window has been assured. The change in the hydrodynamic diameter in the DLS measurement is consistent with the loss of the hydration layer (see below).

The change in hydration number $\left(n_{\mathrm{h}}\right)$, i.e., the number of water molecules associated per SDS head group, obtained from ultrasound velocity measurements is plotted against temperature in Figure 1b. The corresponding values are given in Table 1. At $293 \mathrm{~K}$, the hydration number obtained is 17.3, which is in good agreement with the reported value of 20 by Kunz et al. ${ }^{31}$ using the dielectric relaxation technique. As evidenced from the figure, the hydration number (hydration layer) gradually decreases with an increase in temperature, signifying SDS head group-bound water getting converted into free water with increasing temperature. The linear decrease of $n_{\mathrm{h}}$ with temperature leads us to employ the energy barrier crossing model in which the stable polar head group-bound water gets energized with an elevation of temperature and crosses the energy barrier in order to get free. We assume a very simplified model in which there exists a Boltzmann kind of distribution between free and bound water following the relation

$$
n_{\mathrm{b}}=n_{\mathrm{f}} \mathrm{e}^{-E^{*} / R T}
$$

where $n_{\mathrm{b}}$ and $n_{\mathrm{f}}$ stand for bound and free water molecules at temperature $T$ and $E^{*}$ is the energy difference between the two types of molecules. With an increase in temperature, the population $n_{\mathrm{b}}$ decreases with an concomitant increase in the $n_{\mathrm{f}}$ population. We further assume that $n_{\mathrm{f}}$ is large compared to $n_{\mathrm{b}}$ and does not change appreciably with respect to $n_{\mathrm{b}}$ (this assumption is further supported from our time-resolved solvation experiments (see below)). Thus $E^{*}$ can be calculated using the following equation,

$$
E^{*}=R\left(\frac{1}{T_{2}}-\frac{1}{T_{1}}\right) \ln \left(\frac{n_{\mathrm{b} 1}}{n_{\mathrm{b} 2}}\right)
$$

where $n_{\mathrm{b} 1}$ and $n_{\mathrm{b} 2}$ are the number of bound water at temperature $T_{1}$ and $T_{2}$, respectively. We calculate $E^{*}$ values for different temperature intervals, and the values vary in the range of $2.7-$ $3.5 \mathrm{kcal} \mathrm{mol}^{-1}$. This energy can be empirically equated to the difference between the bound and free states of water at the micellar surface. The value obtained herein is in close agreement to that obtained by $\mathrm{Pal}$ et al. ${ }^{5,10}$ using molecular dynamics (MD) simulation studies.

Figure 2a depicts the fluorescence spectra of DCM, excited at $409 \mathrm{~nm}$, in $50 \mathrm{mM} \mathrm{SDS}$ at 298 and $348 \mathrm{~K}$. The emission peak, which is obtained at $623 \mathrm{~nm}$ at $298 \mathrm{~K}$, is slightly $(\sim 2-3$
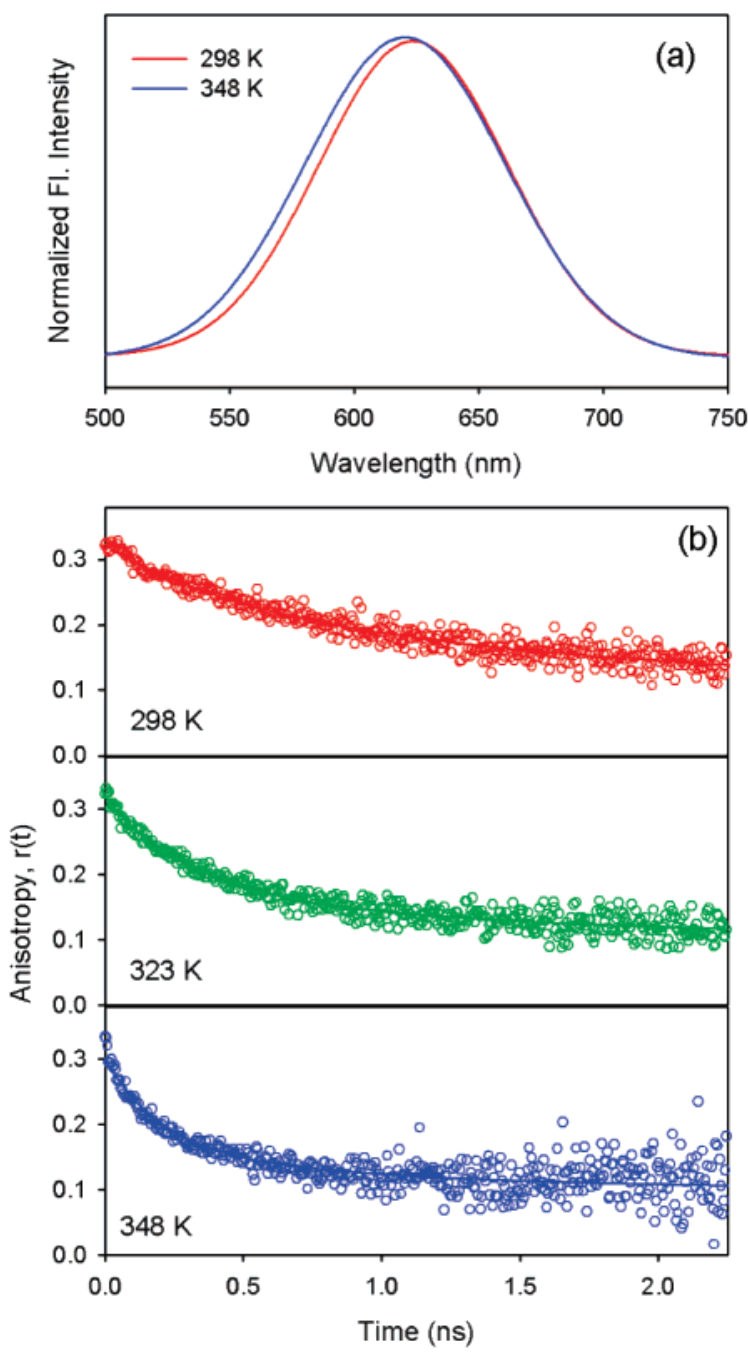

Figure 2. (a) Emission spectra of DCM in $50 \mathrm{mM}$ SDS micelles at 298 and $348 \mathrm{~K}$. The excitation wavelength is $409 \mathrm{~nm}$. (b) Fluorescence anisotropy decay, $r(t)$, of DCM in $50 \mathrm{mM}$ SDS micelles at $620 \mathrm{~nm}$ at 298, 323, and $348 \mathrm{~K}$.

TABLE 2: Fluorescence Anisotropy, $r(t)$, and Decay Parameters of DCM in $50 \mathrm{mM}$ SDS at Different Temperatures

\begin{tabular}{ccccc}
\hline temperature $(\mathrm{K})$ & $r_{\mathrm{o}}$ & offset & $\tau_{\mathrm{r} 1}(\mathrm{~ns})$ & $\tau_{\mathrm{r} 2}(\mathrm{~ns})$ \\
\hline 298 & 0.32 & 0.10 & $0.40(23 \%)$ & $1.6(45 \%)$ \\
323 & 0.32 & 0.11 & $0.28(40 \%)$ & $1.2(27 \%)$ \\
348 & 0.30 & 0.10 & $0.20(46 \%)$ & $1.0(20 \%)$
\end{tabular}

$\mathrm{nm}$ ) blue-shifted upon increasing the temperature to $348 \mathrm{~K}$. Such an insignificant blue shift of the probe indicates that the microenvironment of the probe does not change appreciably with an increase in temperature. It is known that DCM is completely insoluble in water and dissolves only in the interfacial layer of the micelles. ${ }^{21}$ Thus, it might be inferred that no significant change in the probe location occurs upon increasing the temperature and it resides at the interface.

Fluorescence anisotropy decay, $r(t)$, of DCM in SDS micelles, measured at $620 \mathrm{~nm}$ at three different temperatures, has been presented in Figure 2b. For all the studied temperatures, $r(t)$ is characterized by a slow decay with the presence of a considerable offset. The decays fit well biexponentially, and the rotational time constants $\left(\tau_{\mathrm{r} 1}\right.$ and $\left.\tau_{\mathrm{r} 2}\right)$ estimated at different temperatures are listed in Table 2 . It is seen from the table that the anisotropy is associated with an offset value of $\sim 0.1$, which perhaps signifies the rotational motion of the micelle that 

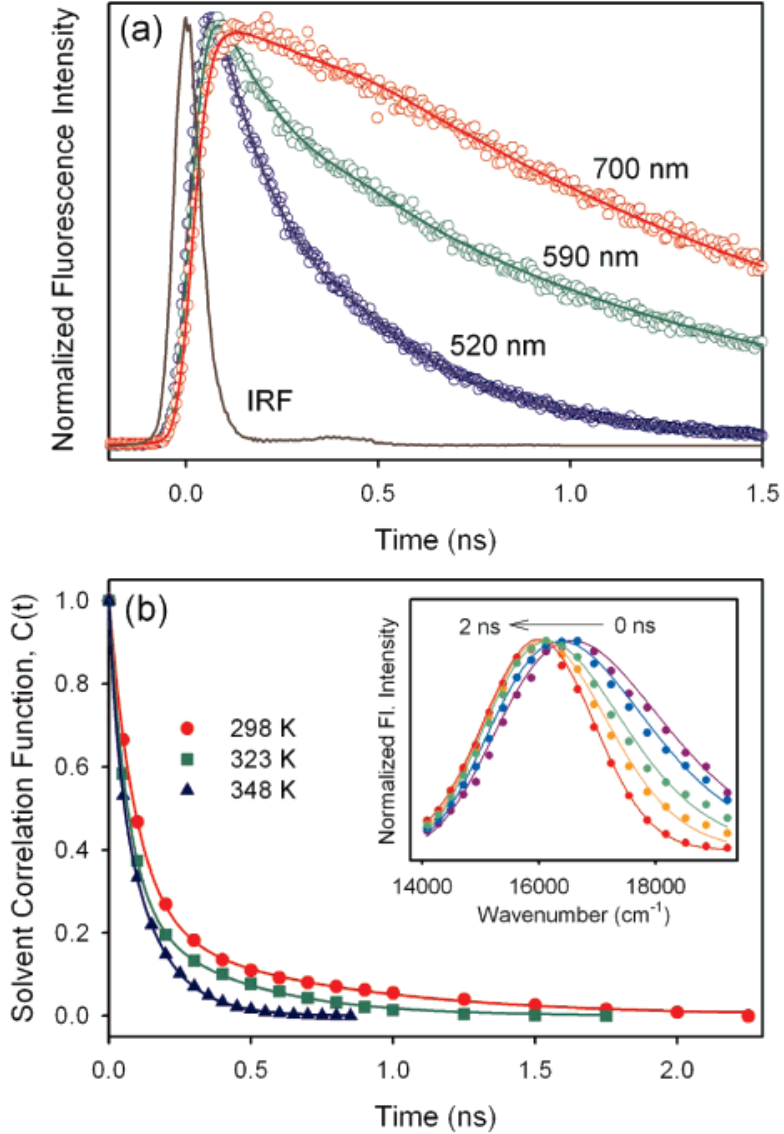

Figure 3. (a) Fluorescence decays of DCM in $50 \mathrm{mM} \mathrm{SDS}$ at $298 \mathrm{~K}$ at 520,590 , and $700 \mathrm{~nm}$. (b) Solvent correlation function, $C(t)$, of DCM in $50 \mathrm{mM}$ SDS at 298, 323, and $348 \mathrm{~K}$. The solid lines denote the best fit to a biexponential decay. Time-resolved emission spectra of DCM in $50 \mathrm{mM}$ SDS at $298 \mathrm{~K}$ are given in the inset.

does not complete within the experimental time window. The decay is associated with two time constants, one on the order of hundreds of picoseconds and the other on the order of $1 \mathrm{~ns}$. The rotational relaxation times obtained in this study are slower than that of picosecond rotational times reported in bulk water. ${ }^{32,33}$ This concludes that the dye experiences much higher microviscosity in micellar systems in comparison to that in bulk water, and thus the probe resides in the interfacial layer of the micelle in all the studied temperatures. The time constants get faster upon increasing temperature which reflects the thermal effect on the microviscosity and consequently to the wobbling rate of the probe and also the increase of free water molecules at the interface. ${ }^{34}$ The presence of offset even at $348 \mathrm{~K}$ confirms the location of the dye within the micellar interface at elevated temperatures. Thus, DCM is highly efficient to probe the dynamics of water located in the interfacial layer at all studied temperatures.

The fluorescence decay transients of DCM in SDS micelles at three representative wavelengths at $298 \mathrm{~K}$ are presented in Figure 3a. It is evidenced from the figure that the decay patterns are strongly wavelength dependent. At $520 \mathrm{~nm}$, the transient is very fast with decay components of $100 \mathrm{ps}(37 \%), 350 \mathrm{ps}(60 \%)$, and $1800 \mathrm{ps}(3 \%)$. The transient becomes slower with an increase in the wavelength, and at the extreme red end (at 700 $\mathrm{nm}$ ), a decay component of $1370 \mathrm{ps}$ along with a rise component of $175 \mathrm{ps}$ are obtained. Similar wavelength dependency is registered for the DCM/SDS system at two other temperatures, with a gradual decrease of an average lifetime with increasing temperature (figures not shown).
TABLE 3: Decay Parameters of $C(t)$ of DCM in $50 \mathrm{mM}$ SDS at Different Temperatures

\begin{tabular}{ccccccc}
\hline temperature $(\mathrm{K})$ & $a_{1}$ & $\tau_{1}(\mathrm{~ns})$ & $a_{2}$ & $\tau_{2}(\mathrm{~ns})$ & $\left\langle\tau_{\mathrm{s}}\right\rangle(\mathrm{ns})$ & $\Delta v\left(\mathrm{~cm}^{-1}\right)$ \\
\hline 298 & 0.77 & 0.10 & 0.23 & 0.68 & 0.24 & 576 \\
323 & 0.80 & 0.08 & 0.19 & 0.48 & 0.16 & 646 \\
348 & 0.39 & 0.04 & 0.60 & 0.14 & 0.10 & 626
\end{tabular}

The time-resolved emission spectra (TRES) of DCM in SDS are constructed at different temperatures following the procedure given in the previous section. Typical TRES spectra for DCM in SDS as obtained at $298 \mathrm{~K}$ have been depicted in the inset of Figure 3b. The time dependent fluorescence Stoke's shifts, as estimated from TRES, are used to construct the normalized spectral shift correlation function or the solvent correlation function $C(t)$ defined as

$$
C(t)=\frac{v(t)-v(\infty)}{v(0)-v(\infty)}
$$

where, $v(0), v(\mathrm{t})$, and $v(\infty)$ are the emission maxima (in $\mathrm{cm}^{-1}$ ) at time $0, t$, and $\infty$. The $v(\infty)$ values have been taken to be the emission frequency beyond which insignificant or no spectral shift is observed. The $C(t)$ function represents the temporal response of the solvent relaxation process occuring around the probe following its photoexcitation and the associated change in the dipole moment.

The $C(t)$ curves obtained at three different temperatures are presented in Figure 3b. All the curves are fitted with good biexponential decays, and the fitted parameters are presented in Table 3. The average solvation time $\left\langle\tau_{\mathrm{s}}\right\rangle$ in the system has been calculated using the equation

$$
\left\langle\tau_{\mathrm{s}}\right\rangle=a_{1} \tau_{1}+a_{2} \tau_{2}
$$

where, $a_{1}$ and $a_{2}$ are relative concentrations corresponding to the solvation times $\tau_{1}$ and $\tau_{2}$, respectively. It is seen from Table 3 that the time constants are on the order of several tens of and several hundreds of picoseconds. Both the components are slower in comparison to the $\sim 1$ ps time scale of bulk water. The component $\tau_{1}$ is comparable to that obtained for the NATATX100 system by Pal et al. ${ }^{35}$ using femtosecond resolved decay. The possibility of a significant missing dynamical Stokes shift cannot be ruled out due to limited time resolution of our experimental setup. Previously, femtosecond-resolved solvation experiments $^{36}$ on a probe C-153 in SDS micelles reported a dynamical Stokes shift of $450 \mathrm{~cm}^{-1}$, which is comparable to those observed in our study $\left(\sim 550-650 \mathrm{~cm}^{-1}\right)$ and estimated to be only $37 \%$ of the total solvation shift. The significantly higher contribution of the faster component in the $C(t)$ decay (Table 3), which might be due to the solvation by free water molecules present at the interfacial stern layer, supports our earlier assumption that $n_{\mathrm{f}}$ is considerably higher than $n_{\mathrm{b}}$. It is evidenced from Table 3 that the magnitudes of both $\tau_{1}$ and $\tau_{2}$ decrease gradually with increases in temperature. This phenomenon is identical to that obtained by Sen et al. ${ }^{12}$ using 4-AP in TX-100 micelles. We assume an Arrhenius dependency of the rate constant and plotted $\ln \left(1 /\left\langle\tau_{\mathrm{s}}\right\rangle\right)$ against $1 / T$. A good linear fit is obtained (Figure 4 ), and the corresponding $E_{\mathrm{a}}$ value is obtained to be $3.5 \pm 0.3 \mathrm{kcal} \mathrm{mol}^{-1}$, which is in excellent agreement with $2.4-4 \mathrm{kcal} \mathrm{mol}^{-1}$ obtained by Pal et al. ${ }^{5}$ and attributed to the barrier energy of the bound to free water transition at the micellar surface. Earlier Sen et al. ${ }^{12}$ obtained a somewhat higher value of $9 \mathrm{kcal} \mathrm{mol}^{-1}$. They attributed this high value of $E_{\mathrm{a}}$ as an energy difference between head group bound water and bulk water, which is on the order of $7-8 \mathrm{kcal}$ $\mathrm{mol}^{-1}$. 


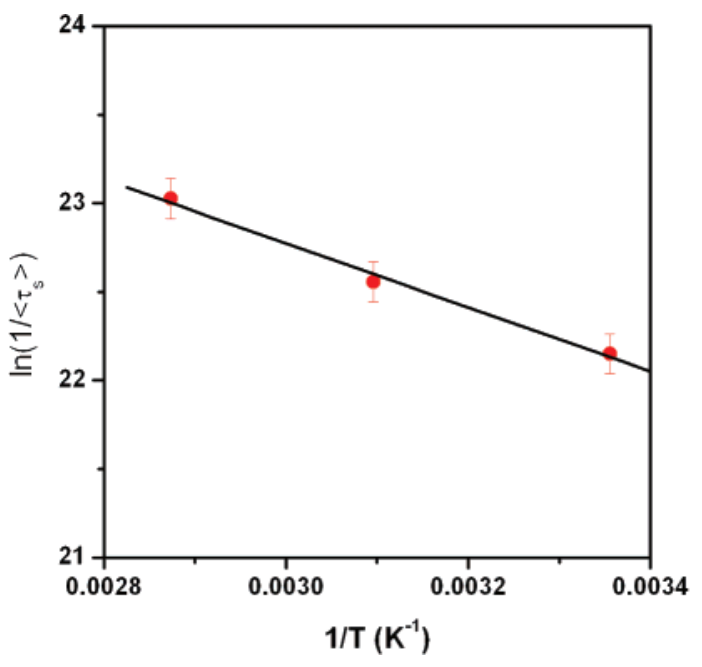

Figure 4. Plot of $\ln \left(1 /\left\langle\tau_{\mathrm{s}}\right\rangle\right)$ against $1 / T$.

The simulation studies of Pal et al. ${ }^{5}$ and Bruce et al. ${ }^{3}$ showed that penetration of water molecules into the SDS micelle is restricted to the head group region, and there exists three different types of water molecules at the micellar interface. Some water molecules (IBW-1) are hydrogen bonded to the polar head group of the surfactant. A few water molecules (IBW-2), which are present in a very low fraction, make two hydrogen bonds with surfactant head groups. The third kind of water molecules (IFW) is not hydrogen-bonded to any polar head group but rather forms hydrogen bonds with other water molecules. All these three kinds of water molecules have energies lower than that of the bulk water. Simulation studies reveal that IBW-1 and IBW-2 are progressively more stable than the IFW species, and the energy difference between IBW-1 and IFW is on the order of $2.4 \mathrm{kcal} \mathrm{mol}^{-1}$. This value is comparable to our estimated $E_{\mathrm{a}}$ value of $3.5 \mathrm{kcal} \mathrm{mol}^{-1}$ in the present study. It can be noted that the probe DCM is insoluble in water and can only sense water molecules that are at the interfacial layer. However, it can be noted that the presence of DCM at the micellar interface induces a perturbation providing a local heterogeneity. Also, when the dye is exposed to a laser, a dipole is induced in the hydrophobic probe and it can migrate to a polar environment. It has been reported earlier that DCM molecules can only migrate within the Stern layer in spite of its induced hydrophilicity. ${ }^{22}$ Therefore, DCM can sense solvation by IBW-1, IBW2 , and IFW molecules in the interfacial stern layer. Thus the $E_{\mathrm{a}}$ value obtained in the present study signifies the activation energy barrier crossing between these interfacial water molecules. On the other hand, Sen et al. ${ }^{12}$ used a hydrophilic probe, 4-AP, which is significantly soluble in bulk water. The probable location of the probe is at the edge of the palisade layer facing the bulk water and thus can only sense the conversion of the interfacially bound water to bulk water, with the corresponding energy barrier on the order of 7-8 $\mathrm{kcal} \mathrm{mol}^{-1}$, which is in close

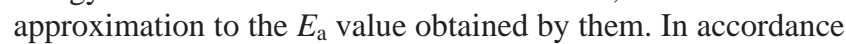
to the argument made by Kumbhakar et al., ${ }^{14}$ the unusual inversion of $\left\langle\tau_{\mathrm{s}}\right\rangle$ with temperature, as obtained by them, has its origin in the probable location of the probe and the combined effect of the change in size and the hydration of the micelle. At a low temperature, the size of the TX-100 micelle is small and the probe can be accessed by bulk water molecules, resulting in fast dynamics. With an increase in temperature, the micelle swells up and the probe is no longer in contact with the bulk water, added with the probability of being partially solvated by the surfactant head groups making the process slower. With a further increase in temperature, the increase is hydration of the micelle perhaps overdoes these effects making the process faster again. The probe used in their study ${ }^{14}$ has its location deeply rooted in the palisade layer in the vicinity of the micellar core and can sense all the associated changes in size and hydration and can report the inversion in $\left\langle\tau_{\mathrm{s}}\right\rangle$. In the present report, we have studied the solvation dynamics within the structural integrity of the micelles, which might have resulted in a gradual decrease in $\left\langle\tau_{\mathrm{s}}\right\rangle$ with temperature. Thus the barrier crossing model might hold good within this condition only. It can also be noted that in the present study, the activation energy of 2.7$3.5 \mathrm{kcal} \mathrm{mol}^{-1}$ calculated from the hydration measurements is in excellent agreement with that obtained from the solvation study $\left(3.5 \mathrm{kcal} \mathrm{mol}^{-1}\right)$. Though the value obtained from the hydration study is only an approximate one, its resemblance with that value from the solvation study leads us to conclude that the change in solvation dynamics with temperature is primarily associated with an activation energy barrier crossing within the framework of the structural integrity of the micelle, in which the rate determining step is the transition between bound and free water molecules.

\section{Conclusions}

The present study confirms the validity of the activation energy barrier crossing model to explain the temperature dependent solvation dynamics of a probe at the micellar surface within the structural integrity of the micelle. Here the fluoroprobe is carefully chosen to reside at the micellar surface. Our results of the activation energy barrier $\left(E_{\mathrm{a}}\right)$ at the micellar surface are consistent with that obtained from MD simulation studies. In the previous studies, the structural integrity of the micelle and the choice of the probes had not been taken into consideration which led to the controversy of the validity of the model. The result obtained from this work concludes that the change in solvation dynamics with temperature is associated with an activation energy barrier crossing within the framework of structural integrity, in which the rate determining step is the transition between bound and free water.

Acknowledgment. We thank DST for financial support (Grant SR/FTP/PS-05/2004).

\section{References and Notes}

(1) Lee, S. H.; Rossky, P. J. J. Chem. Phys. 1994, 100, 3334-3345.

(2) Vajda, S.; Jimenez, R.; Rosenthal, S. J.; Filder, V.; Fleming, G. R.; Castner, E. W., Jr. J. Chem. Soc., Faraday Trans. 1995, 91, 867-873.

(3) Bruce, C. D.; Senapati, S.; Berkowitz, M. L.; Perera, L.; Forbes, M. D. E. J. Phys. Chem. B 2002, 106, 10902-10907.

(4) Pal, S.; Balasubramaian, S.; Bagchi, B. J. Chem. Phys. 2002, 117, $2852-2859$

(5) Pal, S.; Balasubramaian, S.; Bagchi, B. J. Phys. Chem. B 2003, $107,5194-5202$. 0613 .

(7) Nandi, N.; Bagchi, B. J. Phys. Chem. B 1997, 101, 10954-10961.

(8) Nandi, N.; Bhattacharyya, K.; Bagchi, B. Chem. Rev. 2000, 100, 2013-2045.

(9) Ghosh Dastidar, S.; Mukhopadhyay, C. Phys. Rev. E: Stat., Nonlinear, Soft Matter Phys. 2004, 70, 061901.

(10) Balasubramanian, S.; Pal, S.; Bagchi, B. Phys. Rev. Lett. 2002, 89,115505 .

(11) Jimenez, R.; Fleming, G. R.; Kumar, P. V.; Maroncelli, M. Nature 1994, 369, 471-473.

(12) Sen, P.; Mukherjee, S.; Halder, A.; Bhattacharyya, K. Chem. Phys. Lett. 2004, 385, 357-361.

(13) Pal, S. K.; Peon, J.; Bagchi, B.; Zewail, A. H. J. Phys. Chem. B 2002, 106, 12376-12395.

(14) Kumbhakar, M.; Goel, T.; Mukherjee, T.; Pal, H. J. Phys. Chem. B 2004, 108, 19246-19254.

(15) Hara, K.; Kuwabara, H.; Kajimoto, O. J. Phys. Chem. A 2001, 105, 7174-7179. 
(16) Kumbhakar, M.; Goel, T.; Nath, S.; Mukherjee, T.; Pal, H. J. Phys. Chem. B 2006, 110, 25646-25655.

(17) Roy, D.; Mondal, S. K.; Sahu, K.; Ghosh, S.; Sen, P.; Bhattacharyya, K. J. Phys. Chem. A 2005, 109, 7359-7364.

(18) Sahu, K.; Mondal, S. K.; Ghosh, S.; Roy, D.; Bhattacharyya, K. J. Chem. Phys. 2006, 124, 124909.

(19) Vincent, M.; Gilles, A.-M.; Li de la Sierra, I. M.; Briozzo, P.; Barzu, O.; Gallay, J. J. Phys. Chem. B 2000, 104, 11286-11295.

(20) Ingram, J. A.; Moog, R. S.; Ito, N.; Biswas, R.; Maroncelli, M. J. Phys. Chem. B 2003, 107, 5926-5932.

(21) Pal, S. K.; Sukul, D.; Mandal, D.; Sen, S.; Bhattacharyya, K. Chem. Phys. Lett. 2000, 327, 91-96.

(22) Sarkar, R.; Shaw, A. K.; Ghosh, M.; Pal, S. K. J. Photochem. Photobiol., B 2006, 83, 213-222.

(23) Bagchi, B.; Biswas, R. Adv. Chem. Phys. 1999, 109, 207-433.

(24) Bockris, J. O. M.; Saluja, P. P. S. J. Phys. Chem. 1972, 76, 21402151.

(25) Rohman, N.; Mahiuddin, S.; Dass, N. N.; Yoo, K. P. Korean J. Chem. Eng. 2002, 19, 679-684.
(26) Lakowicz, J. R. Principles of Fluorescence Spectroscopy; Kluwer Academic/Plenum: New York, 1999.

(27) Horng, M. L.; Gardecki, J. A.; Papazyan, A.; Maroncelli, M. J. Phys. Chem. 1995, 99, 17311-17337.

(28) O'Connor, D. V.; Philips, D. Time Correlated Single Photon Counting; Academic Press: London, 1984.

(29) Shaw, A. K.; Pal, S. K. J. Photochem. Photobiol., B 2007, 86, 199-206.

(30) Streletzky, K.; Phillies, G. J. D. Langmuir 1995, 11, 42-47.

(31) Fernandez, P.; Schrodle, S.; Buchner, R.; Kunz, W. ChemPhysChem 2003, 4, 1065-1072.

(32) Shaw, A. K.; Sarkar, R.; Pal, S. K. Chem. Phys. Lett. 2005, 408, $366-370$.

(33) Shaw, A. K.; Pal, S. K. J. Phys. Chem. B 2007, 111, 4189-4199.

(34) Maiti, N. C.; Krishna, M. M. G.; Britto, P. J.; Periasamy N. J. Phys. Chem. B 1997, 101, 11051-11060.

(35) Pal, S. K.; Peon, J.; Zewail, A. H. Proc. Natl. Acad. Sci. U.S.A. 2002, 99, 15297-15302.

(36) Sahu, K.; Mondal, S. K.; Ghosh, S.; Roy, D.; Sen, P.; Bhattacharyya, K. J. Phys. Chem. B 2006, 110, 1056-1062. 